\title{
Some Characterizations of Regular and Semisimple $\Gamma$-Rings
}

Xueling MA and JiAnMing ZHAN*

Department of Mathematics, Hubei Institute for Nationalities, Enshi, Hubei Province, 445000, China

e-mail : zjmhbmy@126.com and zhanjianming@hotmail.com

Young BAE Jun

Department of Mathematics Education, Gyeongsang National University, Chinju 660-701, Korea

e-mail : skywine@gmail.com

ABSTRACT. Some characterizations of regular $\Gamma$-rings are described by means of fuzzy ideals. The concepts of fuzzy interior ideals in $\Gamma$-rings and semisimple $\Gamma$-rings are introduced. Some characterizations of semisimple $\Gamma$-rings are investigated by means of fuzzy interior ideals.

\section{Introduction}

As is well known, algebraic structures play a prominent role in mathematics with wide ranging applications in many disciplines such as theoretical physics, computer sciences, control engineering, information sciences, coding theory, topological spaces and so on. This provides sufficient motivations to researchers to review various concepts and results from the realm of abstract algebras in the broader framework of fuzzy setting $[2,8,10,11,15,16]$.

The concept of $\Gamma$-rings is introduced by Barnes [1]. After that, this concept was discussed further by some researchers. The notion of fuzzy ideals in a $\Gamma$-ring was introduced by Jun and Lee in $[6,7]$. They studied some preliminary properties of fuzzy ideals of $\Gamma$-rings. Later in [4] Jun and Hong defined normalized fuzzy ideals and fuzzy maximal ideals in $\Gamma$-rings and studied them. Jun [5] defined fuzzy prime ideal of a $\Gamma$-ring and obtained a number of characterizations for a fuzzy ideal to be a fuzzy prime ideal. Further, Öztürk et al. [12, 13] gave some characterizations of Artinian and Noetherian $\Gamma$-rings. In particular, Dutta and Chanda [3], studied the structures of the set of fuzzy ideals of a $\Gamma$-ring and characterize $\Gamma$-fields, Noetherian $\Gamma$-rings, etc. with the help of fuzzy ideals via operator rings of $\Gamma$-rings. In 1987,

* Corresponding Author.

Received Februry 18, 2010; accepted August 5, 2010.

2000 Mathematics Subject Classification: 16P99, 16D25.

Key words and phrases: (regular, semisimple) $\Gamma$-ring, (interior) ideal, fuzzy ideal, fuzzy interior ideal. 
Kyuno et al. [9] introduced the concept of $\Gamma$-rings and investigated some related properties. Further, Rashid et al. defined another type of regular $\Gamma$-rings. These $\Gamma$-rings are more significant and more general than that of Kyuno. They have shown that the class of all regular $\Gamma$-rings is a radical class.

This present paper is organized as follows. In Section 2, we recall some basic definitions and results of rings. Some characterization theorems of fuzzy ideals of a regular $\Gamma$-ring will be discussed in Section 3. In Section 4, we describe the semisimple $\Gamma$-rings by using fuzzy interior ideals.

\section{Preliminaries}

Definition 2.1([1]). Let $M$ and $\Gamma$ be two additive abelian groups. Then $M$ is called $a \Gamma$-ring if the following conditions are satisfied for all $a, b, c \in M$ and for all $\alpha, \beta, \gamma \in \Gamma$ :

(i) $a \alpha b \in M$;

(ii) $(a+b) \alpha c=a \alpha c+b \alpha c, a(\alpha+\beta) b=a \alpha b+a \beta b, a \alpha(b+c)=a \alpha b+a \alpha c$;

(iii) $a \alpha(b \beta c)=(a \alpha b) \beta c$.

Throughout this paper $M$ denotes a $\Gamma$-ring, and $0_{M}$ denotes the zero element of $M$. We shall write $a \wedge b$ for $\min \{a, b\}$ and $a \vee b$ for $\max \{a, b\}$, where $a$ and $b$ are any two real numbers.

Definition 2.2([1]). A subset $A$ of $M$ is called a left (resp., right) ideal of $M$ if $A$ is an additive subgroup of $M$ and

$M \Gamma A=\{x \alpha y \mid x, y \in M, \alpha \in \Gamma\} \quad($ resp., $A \Gamma M)$ is contained in $A$.

Note that $A$ is called an ideal of $M$ if it is both a left ideal and a right ideal.

A subset $A$ of $M$ is called a interior ideal of $M$ if $A$ is an additive closed subgroup with $M \Gamma A \Gamma M \subseteq A$.

We next state some fuzzy logic concepts. Recall that a fuzzy set is a function $\mu: M \rightarrow[0,1]$. For any $A \subseteq M$, the characteristic function of $A$ is denoted by $\chi_{A}$.

Definition 2.3([6], [7]). A fuzzy set $\mu$ of $M$ is called a fuzzy left (resp., right) ideal of $M$ if for all $x, y \in M$, the following conditions are satisfied:

(F1) $\mu(x-y) \geq \mu(x) \wedge \mu(y)$,

(F2) $\mu(x \alpha y) \geq \mu(y)$ (resp., $\mu(x \alpha y) \geq \mu(x)$ ).

Note that $A$ is called a fuzzy ideal of $M$ if it is both a fuzzy left ideal and a fuzzy right ideal.

Definition 2.4([5]). For any two fuzzy sets $\mu$ and $\nu$ of $M$ and $\gamma \in \Gamma$, then the $\Gamma$-product of $\mu$ and $\nu$ is defined by

$$
(\mu \Gamma \nu)(x)=\bigvee_{x=y \gamma z}(\mu(y) \wedge \nu(z))
$$

and $(\mu \Gamma \nu)(x)=0$ if $x$ cannot be expressed as $x=y \gamma z$. 
Lemma 2.5([5]). If $\mu$ and $\nu$ are fuzzy left (right) ideals of $M$, then so is $\mu \cap \nu$, where $\mu \cap \nu$ is defined by

$(\mu \cap \nu)(x)=\mu(x) \wedge \nu(x), \forall x \in M$.

Moreover, if $\mu$ and $\nu$ are a fuzzy right ideal and a fuzzy left ideal of $M$, respectively, then $\mu \Gamma \nu \subseteq \mu \cap \nu$.

The following is obvious and we omit the proof.

Proposition 2.6. Let $A, B \subseteq M$. Then we have

(1) $A \subseteq B \Leftrightarrow \chi_{A} \subseteq \chi_{B}$;

(2) $\chi_{A} \cap \chi_{B}=\chi_{A \cap B}$

(3) $\chi_{A} \Gamma \chi_{B}=\chi_{A \Gamma B}$.

\section{Characterizations of regular $\Gamma$-rings}

We characterize the regular $\Gamma$-rings in this Section by fuzzy ideals.

Definition 3.1([9]). A $\Gamma$-ring $M$ is said to be regular if for each $x \in M$, there exist $a \in M$ and $\alpha, \beta \in \Gamma$ such that $x=x \alpha a \beta x$.

Lemma 3.2. If $A$ and $B$, are respectively, a right and a left ideal of a $\Gamma$-ring $M$, then $A \Gamma B \subseteq A \cap B$.

Proof. Let $x \in A \Gamma B$, then there exist $a \in A, b \in B$ and $\alpha \in \Gamma$ such that $x=a \alpha b$. Since $A$ is a right ideal of $M$, then $a \alpha b \in M$, that is, $x \in A$. Similarly, $x \in B$. Thus, $x \in A \cap B$. Hence $A \Gamma B \subseteq A \cap B$.

Lemma 3.3. $A \Gamma$-ring $M$ is regular if and only if for any right ideal $A$ and any left ideal $B$, we have $A \Gamma B=A \cap B$.

Proof. Set $x \in A \cap B$, then $x \in A$ and $x \in B$. Since $M$ is regular, then there exist $\alpha, \beta \in \Gamma$ and $a \in M$ such that $x=x \alpha a \beta x$. Since $A$ is a right ideal of $M$, then $x \alpha a \in A$, and so $(x \alpha a) \beta x \in A \Gamma B$, which implies $A \cap B \subseteq A \Gamma B$. By Lemma 3.2, $A \Gamma B=A \cap B$.

Conversely, set $x \in M$, then $x \Gamma M$ and $M \Gamma x$ is a right ideal and a left ideal of $M$, respectively. Then $x \in x \Gamma M \cap M \Gamma x=x \Gamma M \Gamma M \Gamma x \subseteq x \Gamma M \Gamma x$. Then there exist $\alpha, \beta \in \Gamma$ and $a \in M$ such that $x=x \alpha a \beta x$. Therefore, $M$ is regular.

Lemma 3.4. A fuzzy set $\mu$ of $M$ is a fuzzy left (resp., right) ideal of $M$ if and only if for all $x, y \in M$ and $\alpha \in \Gamma$, it satisfies (F1) and (F3) $\chi_{M} \Gamma \mu \subseteq \mu$ (resp., $\left.\mu \Gamma \chi_{M} \subseteq \mu\right)$.

Proof. We only prove the case for fuzzy left ideals. The case for fuzzy right ideals can be similarly proved.

Let $\mu$ be a fuzzy left ideal of $M$, for any $x \in M$, if $\left(\chi_{M} \Gamma \mu\right)(x)=0$, it is clear that $\left(\chi_{M} \Gamma \mu\right)(x) \leq \mu(x)$. Otherwise, there exist $u, v \in M$ and $\gamma \in \Gamma$ such that $x=u \gamma v$. 
Thus,

$$
\begin{aligned}
\left(\chi_{M} \Gamma \mu\right)(x) & =\bigvee_{x=u \gamma v}\left(\chi_{M}(u) \wedge \mu(v)\right) \\
& =\bigvee_{x=u \gamma v}(\mu(v)) \\
& \leq \bigvee_{x=u \gamma v}(\mu(u \gamma v)) \\
& =\bigvee_{x=u \gamma v}(\mu(x)) \\
& =\mu(x),
\end{aligned}
$$

which implies, $\chi_{M} \Gamma \mu \subseteq \mu$. This proves (F3) holds.

Conversely, assume that the given conditions hold. For any $x, y \in M$ and $\alpha \in \Gamma$, we find

$$
\begin{aligned}
\mu(x \alpha y) & \geq\left(\chi_{M} \Gamma \mu\right)(x \alpha y) \\
& =\bigvee_{x \alpha y=u \gamma v}\left(\chi_{M}(u) \wedge \mu(v)\right) \\
& \geq \chi_{M}(x) \wedge \mu(y) \\
& =\mu(y) .
\end{aligned}
$$

This proves that $\mu$ is a fuzzy left ideal of $M$.

Now, we characterize the regular $\Gamma$-rings by fuzzy ideals.

Thoerem 3.5. $A$-ring $M$ is regular if and only if for any fuzzy right ideal $\mu$ and fuzzy left ideal $\nu$, we have $\mu \cap \nu=\mu \Gamma \nu$.

Proof. Let $M$ be a regular $\Gamma$-ring, $\mu$ a fuzzy right ideal and $\nu$ a fuzzy left ideal. Then by Lemma 3.4, we have

$\mu \Gamma \nu \subseteq \mu \Gamma \chi_{M} \subseteq \mu$ and $\mu \Gamma \nu \subseteq \chi_{M} \Gamma \nu \subseteq \nu$. Thus, $\mu \Gamma \nu \subseteq \mu \cap \nu$.

For any $x \in M$, there exist $a \in M$ and $\alpha, \beta \in \Gamma$ such that $x=x \alpha a \beta x$ since $M$ is regular. Thus

$$
\begin{aligned}
(\mu \Gamma \nu)(x) & =\bigvee_{x=u \gamma v}(\mu(u) \wedge \nu(v)) \\
& \geq \mu(x \alpha a) \wedge \nu(x) \\
& \geq \mu(x) \wedge \nu(x) \\
& =(\mu \cap \nu)(x) .
\end{aligned}
$$

Thus $\mu \cap \nu \subseteq \mu \Gamma \nu$, hence $\mu \cap \nu=\mu \Gamma \nu$.

Conversely, let $A$ and $B$ be, respectively a right and a left ideal of $M$. Then, it is easy to see that their characteristic functions $\chi_{A}$ and $\chi_{B}$ are, respectively, a fuzzy right ideal and a fuzzy left ideal. Thus, by Proposition 2.6, we have

$\chi_{A \Gamma B}=\chi_{A} \Gamma \chi_{B}=\chi_{A} \cap \chi_{B}=\chi_{A \cap B}$.

Thus, by Proposition 2.6, $A \Gamma B=A \cap B$. It follows from Lemma 3.3 that $M$ is regular.

\section{Characterizations of semisimple $\Gamma$-rings}

In this Section, we first introduce the concept of fuzzy interior ideals in $\Gamma$-rings. 
By this kind of fuzzy ideals, we characterize the semisimple $\Gamma$-rings.

Definition 4.1. A fuzzy set $\mu$ of $M$ is called a fuzzy interior ideal of $M$ if for all $x, y, z \in M$ and $\alpha, \beta \in \Gamma$, it satisfies (F1) and

(F4) $\mu(x \alpha y) \geq \mu(x) \wedge \mu(y)$,

(F5) $\mu(x \alpha y \beta z) \geq \mu(y)$.

The following theorem is obvious.

Thoerem 4.2. Every fuzzy ideal of $M$ is a fuzzy interior ideal.

Example 4.3. If $G$ and $H$ are additive abelian groups and $M=H o m(G, H), \Gamma=$ $\operatorname{Hom}(H, G)$, then $M$ is a $\Gamma$-ring with the operations pointwise addition and compositions of homomorphisms.

Define a fuzzy set $\mu$ of $M$ by $\mu\left(0_{M}\right)=0.5$ and $\mu(f)=0.3, \forall f\left(\neq 0_{M}\right) \in M$. By routine calculations, $\mu$ is a fuzzy interior ideal of $M$.

Definition 4.4. A subset $A$ of $M$ is called $\Gamma$-idempotent if $A=A \Gamma A$.

$A \Gamma$-ring $M$ is called semisimple if every ideal of $M$ is $\Gamma$-idempotent.

Lemma 4.5. Let $M$ be a $\Gamma$-ring. Then the following are equivalent:

(1) $M$ is semisimple;

(2) $a \in M \Gamma a \Gamma M \Gamma a \Gamma M$, for all $a \in M$;

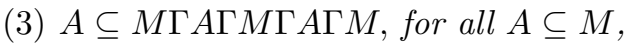

Proof. (1) $\Rightarrow(2)$ Let $M$ be a semisimple $\Gamma$-ring. For any $a \in M$, we have $a \Gamma M+$ $M \Gamma a+M \Gamma a \Gamma M+N a$, where $N=\{0,1,2, \ldots\}$, is the principle ideal of $M$ generated by $a$. Thus,

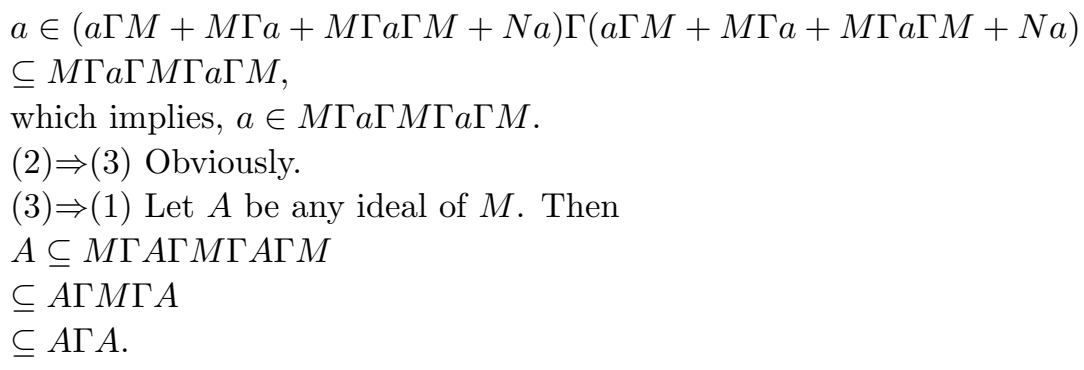

The converse inclusion always holds. Thus, $A=A \Gamma A$. Therefore, $M$ is semisimple.

Next, we discuss the relationship between fuzzy ideals and fuzzy interior ideas in semisimple $\Gamma$-rings.

Thoerem 4.6. Let $M$ be a semisimple $\Gamma$-ring and $\mu$ any fuzzy set of $M$. Then $\mu$ is a fuzzy ideal if and only if it is a fuzzy interior ideal.

Proof. If $\mu$ is a fuzzy ideal of $M$, then by Theorem $4.2, \mu$ is a fuzzy interior ideal. 
Conversely, suppose $\mu$ is a fuzzy interior ideal of $M$. Set $x, y \in M$ and $\alpha \in \Gamma$. Since $M$ is semisimple, by Lemma 4.5 , there exist $a, b, c, d \in M$ and $\beta_{i} \in \Gamma(i=$ $1,2,3,4,5)$ such that $x=a \beta_{1} x \beta_{2} b \beta_{3} c \beta_{4} x \beta_{5} d$, and so $x \alpha y=a \beta_{1} x \beta_{2} b \beta_{3} c \beta_{4} x \beta_{5} d \alpha y$.

Thus, $\mu(x \alpha y)$

$=\mu\left(\left(a \beta_{1} x \beta_{2} b \beta_{3} c\right) \beta_{4} x \beta_{5}(d \alpha y)\right)$

$\geq \mu(x)$.

This proves that $\mu$ is a fuzzy right ideal of $M$. Similarly, we can prove that $\mu$ is a fuzzy left ideal of $M$. Therefore, $\mu$ is a fuzzy ideal of $M$.

Finally, we give a characterization of semisimple $\Gamma$-rings.

Thoerem 4.7. $A \Gamma$-ring $M$ is semisimple if and only if for any fuzzy interior ideals $\mu$ and $\nu$, we have $\mu \cap \nu=\mu \Gamma \nu$.

Proof. Let $M$ be a semisimple $\Gamma$-ring. If $\mu$ and $\nu$ are fuzzy interior ideals of $M$, then by Theorem 4.6, $\mu$ and $\nu$ are fuzzy ideals of $M$. Thus, $\mu \Gamma \nu \subseteq \mu \Gamma \chi_{M} \subseteq \mu$ and $\mu \Gamma \nu \subseteq \chi_{M} \Gamma \nu \subseteq \nu$. Thus, $\mu \Gamma \nu \subseteq \mu \cap \nu$.

For any $x \in M$ and $\alpha \in \Gamma$, since $M$ is semisimple, by Lemma 4.5 , there exist $a, b, c, d \in M$ and $\beta_{i} \in \Gamma(i=1,2,3,4,5)$ such that $x=a \beta_{1} x \beta_{2} b \beta_{3} c \beta_{4} x \beta_{5} d$.

Thus, we have

$$
\begin{aligned}
(\mu \Gamma \nu)(x) & =\bigvee_{x=u \gamma v}(\mu(u) \wedge \nu(v)) \\
& \geq \mu\left(a \beta_{1} x \beta_{2} b\right) \wedge \nu\left(c \beta_{4} x \beta_{5} d\right) \\
& \geq \mu(x) \wedge \nu(x) \\
& =(\mu \cap \nu)(x),
\end{aligned}
$$

i. e., $\mu \cap \nu \subseteq \mu \Gamma \nu$, whence $\mu \cap \nu=\mu \Gamma \nu$.

Conversely, let $A$ be any ideal of $M$, then it is an interior ideal. Thus, its characteristic functions $\chi_{A}$ is a fuzzy interior ideal. Thus, we have

$\chi_{A}=\chi_{A} \cap \chi_{A}=\chi_{A} \Gamma \chi_{A}=\chi_{A \Gamma A}$,

which implies, $A=A \Gamma A$. Thus, $M$ is semisimple.

\section{Conclusions}

In the study of the structure of a fuzzy algebraic system, we notice that the (fuzzy) ideals with special properties always play an important role. In this paper, we describe some characterizations of regular $\Gamma$-rings by means of fuzzy ideals. The concepts of fuzzy interiors of $\Gamma$-rings and semisimple $\Gamma$-rings are introduced. Some characterizations of semisimple $\Gamma$-rings are investigated by means of fuzzy interior ideals.

We hope that the research along this direction can be continued, and in fact, some results in this paper have already constituted a platform for further discussion concerning the future development of $\Gamma$-rings. In our future study of fuzzy structure of $\Gamma$-rings, may be the following topics should be considered:

(1) To describe soft $\Gamma$-rings and its applications; 
(2) To establish three isomorphism theorems of soft $\Gamma$-rings;

(3) To investigate rough fuzzy ideals in $\Gamma$-rings.

Acknowledgements. The research is partially supported by the National Natural Science Foundation of China (60875034); the Natural Science Foundation of Education Committee of Hubei Province, China (D20092901; Q20092907) and the Natural Science Foundation of Hubei Province, China (2009CDB340).

\section{References}

[1] W. E. Barnes, On the Г-rings of Nobusawa, Pacific J. Math., 18(1966), 411-422.

[2] B. Davvaz, P. Corsini, Generalized fuzzy hyperideals of hypernear-rings and many valued implications, Journal of Intelligent and Fuzzy Systems, 17(2006), 241-251.

[3] T. K. Dutta, T. Chanda, Structures of fuzzy ideals of $\Gamma$-rings, Bull. Malays. Math. Sci. Soc., 28(1)(2005), 9-15.

[4] S. M. Hong, Y. B. Jun, A note on fuzzy ideals in gamma-rings, Bull. Honam Math. Soc., 12(1995), 39-48.

[5] Y. B. Jun, On fuzzy prime ideals of $\Gamma$-rings, Soochow J. Math., 21(1)(1995), 41-48.

[6] Y. B. Jun, C. Y. Lee, Fuzzy Г-rings, Pusan Kyongnan Math. J. (presently, East Asian Math. J.), 8(2)(1992), 163-170.

[7] Y. B. Jun, C. Y. Lee, Fuzzy prime ideals of $\Gamma$-rings, Pusan Kyongnan Math. J. (presently, East Asian Math. J.), 9(1)(1993), 105-111.

[8] H. V. Kumbhojkar, M. S. Bapat, Not-so-fuzzy fuzzy ideals, Fuzzy Sets and Systems, 37(1990), 237-243.

[9] S. Kyuno, N. Nobusawa, N. B. Sith, Regular gamma rings, Tsykuba J. Math., 11(2)(1987), 371-382.

[10] X. Ma, J. Zhan, Generalized fuzzy h-bi-ideals and h-quasi-ideals of hemirings, Inform. Sci., 179(2009), 1249-1268.

[11] T. K. Mukherjee, M. K. Sen, On fuzzy ideals of a ring (I), Fuzzy Sets and Systems, 21(1987), 99-104.

[12] M. A. Öztürk, M. Uckum, Y. B. Jun, Fuzzy ideals in Gamma-rings, Turk. J. Math., 27(2003), 369-374.

[13] M. A. Öztürk, M. Uckum, Y. B. Jun, Characterizations of Artinian and Noetherian gamma-rings in terms of fuzzy ideals, Turk. J. Math., 27(2002), 199-205.

[14] M. M. Rashid, A. C. Paul, Regular gamma rings, Southeast Asian Bull. Math., 31(2007), 933-947.

[15] J. Zhan, W. A. Dudek, Fuzzy h-ideals of hemirings, Inform. Sci., 177(2007), 876-886.

[16] J. Zhan, B. Davvaz, K. P. Shum, Generalized fuzzy hyperideals of hyperrings, Comput. Math. Appl., 56(2008), 1732-1740. 